tions of CK-MB that are typically less than expected given the extent of left ventricular dysfunction (echocardiography) will aid differential diagnosis. ${ }^{5}$ The frequency with which arrhythmias are encountered in these patients mandates continuous ECG monitoring during the first days following the acute event.

1 Byer E, Ashman R, Toth L. Electrocardiograms with large, upright $\mathrm{T}$ waves and long QT intervals. $A m \mathcal{F}$ Heart 1947;33:796-806.

2 Kono T, Morita H, Kuroiwa T, Onaka H, Takatsuka H, Fujiwara A. Left ventricular wall motion abnormalities in patients with subarachnoid hemorrhage: neurogenic patients with subarachnoid hemorrhage: neurogen

3 Samra SK, Kroll DA. Subarachnoid hemorrhage and intraoperative electrocardiographic changes simulating myocardial ischemia-anaesthesiologist's dilemma. Anesth Analg 1985;64:86-9.

\section{Final diagnosis}

Subarachnoid haemorrhage causing ECG abnormalities.

Keywords: subarachnoid haemorrhage; electrocardiography; coronary disease; arrhythmia

4 Manninen PH, Ayra B, Gelb AW, Pelz D. Association between electrocardiographic abnormalities and intracranial blood in patients following acute subarachnoid hemorrhage. f Neurosurg Anesthesiol 1995;7:12-6.

5 Mayer SA, Swarup R. Neurogenic cardiac injury after subarachnoid hemorrhage. Curr Opin Anaesthesiol 1996;9:356-

\title{
An abdominal mass following a road traffic accident
}

\author{
J W F Catto, F Hinson, D J Alexander
}

An 18-year-old woman presented to her local Emergency department three weeks after a road accident. As an unrestrained backseat passenger she had been ejected from a car rolling over at $60 \mathrm{mph}$. Initially she was well and she was discharged from the local hospital after a period of observation. During the following weeks she had developed abdominal and back pain associated with anorexia. On examination she was found to have a tachycardia (108 beats $/ \mathrm{min})$ and a tender mass in the epigastrium. Following blood tests showing hyperamylasaemia $(1271 \mathrm{IU} / \mathrm{ml})$, and anaemia $(9.8 \mathrm{~g} / \mathrm{dl}$ ), she had an abdominal computed tomography (CT) scan (figure).

\section{Department of General Surgery, York District Hospital, York, North Yorkshire, UK J W F Catto \\ F Hinson \\ D J Alexander \\ Correspondence to $\mathrm{Dr}$ Catto, 20 Avondale Court, Shadwell Lane, Moortown, Leeds LS17 6DT, UK}

Accepted 10 March 1998

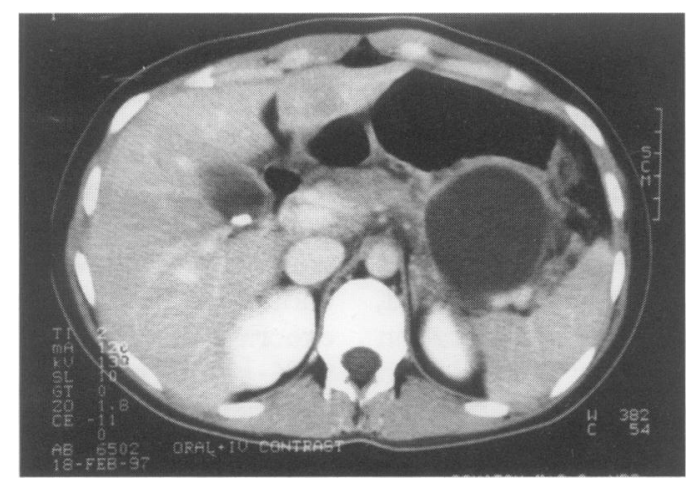

\section{Questions}

1 Comment on the history and CT scan.

2 What is the injury and the mechanism?

3 What further investigations would you do?

4 How would you manage this patient?

5 What other complications might this patient develop?

Figure Abdominal CT scan 
Answers

QUESTION 1

Any high velocity accident should always give rise to a suspicion of serious injury. The CT scan demonstrates a pancreatic pseudocyst from a distal pancreatic duct rupture. The pancreas is damaged in $0.2-6 \%$ of cases of abdominal trauma, ${ }^{1}$ while $50-98 \%$ of pancreatic injuries ${ }^{2}$ occur in association with other visceral injury and therefore may be discovered at laparotomy. Isolated pancreatic injuries are notoriously difficult to diagnose. The retroperitoneal position of the pancreas may lead to few abdominal signs. It is well documented for isolated pancreatic injuries to present after the event. The time of presentation varies from weeks after, with abdominal symptoms, to years later with chronic pancreatitis.

\section{QUESTION 2}

The pseudocyst has arisen following damage to the pancreatic ductal system. Transection of the distal main duct was found at laparotomy. The pancreas is fixed in the retroperitoneum and during an accident it may become compressed onto the vertebral column before shearing forces damage its architecture.

\section{QUESTION 3}

Serum amylase at the time of injury is useful but unreliable, only $60 \%$ of acute pancreatic injuries having hyperamylasaemia and only $8 \%$ of abdominal trauma with hyperamylasaemia having pancreatic injury. ${ }^{34}$ Immediate CT and peritoneal lavage have been shown to be helpful but unreliable. ${ }^{24}$ Diagnosis can be made by endoscopic retrograde cholangiopancreatography (or intra-operative pancreatography), delayed CT or by laparotomy, therefore a high degree of suspicion is needed.

\section{QUESTION 4}

If there is any suspicion of serious intraabdominal pathology, initial resuscitation is the priority. When other viscera or vessels are damaged, a state of shock may be present. Laparotomy is indicated if there is a penetrating wound or a significant bleeding source. At laparotomy several viscera may be damaged, and it can be easy to miss a pancreatic laceration or abrasion unless specifically looked for. Indeed, most deaths in patients with pancreatic damage are from associated injuries. ${ }^{12}$

Patients with isolated pancreatic injuries are usually haemodynamically stable. The integrity of the main pancreatic duct is the major factor in dictating a treatment regimen. Lucas ${ }^{5}$ thus classified pancreatic injuries accordingly (box). Pancreatography is an important step in planning treatment strategy.

Grade 1 and 2 injuries may be managed conservatively with wide-bore percutaneous

1 Leppaniemi A, Haapiaanen R, Kiviluoto T, Lempinen $M$. Pancreatic trauma: acute and late manifestations. $B r \mathcal{F}$ Surg 988;75:165-7.

2 Wilson RH, Moorehead RJ. Current management of trauma to the pancreas. Br $\mathcal{F}$ Surg 1991;78:1196-202.

3 Boulnger BR, Milzman DP, Rosati C, Rodriguez A. The 3 Boulnger BR, Milzman DP, Rosati C, Rodriguez A. The trauma. Can $\mathcal{F}$ Surg 1993;36:63-9.

\section{Modified Lucas classification of} pancreatic trauma

- Class 1: Contusion or peripheral laceration with minimal parenchymal damage; main pancreatic duct intact

- Class 2: Major laceration, perforation or transection of body or tail with or without duct injury

- Class 3: Severe crush, perforation or transection of the pancreatic head with or without duct injury

- Class 4: Combined pancreaticoduodenal injury:

(a) minor pancreatic injury

(b) severe pancreatic injury with duct disruption

drainage of the leaking pancreatic secretions. Administration of octreotide is used to minimise pancreatic secretion. Should complications arise or drainage fail to work alone, then distal pancreatectomy is advocated rather than repair of the duct.

Grade 3 and 4 injuries often require operative treatment. Injuries to the head and proximal main duct should be treated by initial drainage and repair of associated viscera (eg, duodenum), unless there is devitalisation to the pancreas which requires resection. Opinions differ as to the solution in proximal main duct rupture surgery. The choices are between conservative drainage, distal pancreatectomy and internal drainage, ie, cystogastrostomy or cystojejunostomy.

\section{QUESTION 5}

Morbidity and mortality will vary with the nature and extent of injury. The initial complications are those of associated injuries leading to haemorrhage, perforation and shock. The commonest complications are fistula and abscess formation, which occur in $10-25 \%$ of cases. ${ }^{4}$ There is an increased risk if other viscera are damaged. Pancreatitis and pseudocysts are also often seen and may be the presenting complaint in isolated injury. Acute pancreatitis following trauma may be severe enough to be life-threatening. Endocrine and exocrine insufficiencies are rarely seen. Over $90 \%$ of the pancreas can be resected before insufficiency is seen.

Mortality rates of $3-10 \%$ have been described in isolated pancreatic injury. Overall mortality in cases of multiple trauma is between $13-31 \%{ }^{1}$

\section{Final diagnosis}

Post-traumatic pancreatic pseudocyst.

Keywords: pseudocyst; trauma; pancreas

4 Jurkovich GJ, Carrico CJ. Pancreatic trauma. Surg Clin North Am 1990;70:575-93.

5 Lucas CE. Diagnosis and treatment of pancreatic and duodenal injury. Surg Clin North Am 1977;57:49-65. 